\title{
Continuous arterial-venous diahemofiltration and continuous veno-venous diahemofiltration in infants and children
}

\author{
Timothy E. Bunchman' ${ }^{1}$ and Raymond A. Donckerwolcke ${ }^{2}$ \\ ${ }^{1}$ C. S. Mott Children's Hospital, The University of Michigan Medical Center, Box 0297-L-2602, 1521 Simpson Road East, Ann Arbor, MI 48109, USA \\ 2 Wilhelmina Children's Hospital, Postbus 18009, 3501 CA Utrecht, The Netherlands
}

Received June 1, 1993; accepted June 15, 1993

\begin{abstract}
Continuous arterial-venous diahemofiltration and continuous veno-venous diahemofiltration [CAVH(D)/CVVH(D)] in the infant and pediatric population is increasingly being utilized in the child needing renal replacement therapy (RRT). Difficulties with infant- and pediatric-specific equipment remains a limitation. The availability of techniques and equipment in this unique population is addressed. Use of this form of RRT as opposed to hemodialysis or peritoneal dialysis is discussed. The decision for CAVH(D) or CVVH(D) remains an individual choice.
\end{abstract}

Key words: Continuous veno-venous diahemofiltration Continuous arterial-venous diahemofiltration - Heparin

\section{Introduction}

This paper addresses the methodology of continuous arterio-venous hemofiltration (CAVH), continuous venovenous hemofiltration (CVVH), continuous arterialvenous diahemofiltration [CAVH(D)], as well as continuous veno-venous diahemofiltration [CVVH(D)]. The focus of this paper is to involve the reader in understanding each specific technique and the methodologies utilized. Indications as well as relative contraindications will be presented with a practical application of each one being discussed.

The indications for these techniques are primarily for ultrafiltration requirements in patients [1-6]. Ultrafiltration utilizing CAVH/CVVH is easily attained with less hemodynamic instability than hemodialysis (HD) or peritoneal dialysis (PD). Clearance (e.g., urea clearance) is more efficiently attained by HD or PD than by CAVH(D) and CVVH(D) [7, 8]. The advantage of CAVH(D) or CVVH(D) over HD is that of its continuous nature as well

Correspondence to: T. E. Bunchman as causing less hemodynamic instability during ultrafiltration, due to isotonic fluid removal in the patient. It has proven to be effective in a variety of conditions associated with acute renal failure as well as adjunct therapy providing clearance in metabolic diseases [9-14]. The advantage of CAVH(D) or CVVH(D) over PD is not only its ease of administration but its decreased risk of complications associated with the peritoneum, such as increased abdominal pressure [15], decrease in ventilation [16], peritoneal plural leaks [17], and peritonitis [18]. Furthermore, in those patients recently having undergone abdominal surgery, $\mathrm{PD}$ is a relative contraindication.

The immediate disadvantage of $\mathrm{CAVH}(\mathrm{D})$ or CVVH(D) is that of the requirement of systemic heparinization. The degree of heparinization is related to the blood flow rate as well as the amount of hemoconcentration that occurs dependent upon the degree of ultrafiltration. The more rapid blood flow rate and the lesser degree of hemoconcentration require less heparinization.

\section{Access-CAVH(D)}

CAVH(D) requires arterial as well as venous access. The size of the individual will determine the access (Table 1). In infants and neonates, CAVH(D) access has been attained by a 3.5- to 5-Fr umbilical arterial catheter (UAC) with return to a 5- to 8.5-Fr umbilical venous catheter (UVC) [11]. Attention to placement of the UVC and UAC catheters is important. The combination of adequate blood flow balanced with the position (and safety) of these catheters is important. Institutional preferences may dictate "high" versus "low" line placement. The advantage of using the UAC and the UVC in the neonate is its ease of cannulation. The disadvantage is that the catheter utilized in this setting has a relatively high resistance. The blood flow rate is directly related to the resistance of the arterial as well as the venous tubing. The resistance within a tube is related to the internal diameter as well as the length [19]. Thus, if one has a relatively small bore yet long catheter, the result is a relatively high resistance circuit causing a 
Table 1. Suggested acute access

\begin{tabular}{lll}
\hline Size & French & Access \\
\hline Neonate & UAC $3.5-5.0 \mathrm{Fr}$ & Umbilical \\
& UVC $5.0-8.5 \mathrm{Fr}$ & \\
or & \\
5.0 Single lumen & $\begin{array}{c}\text { Femoral vein } \\
\text { or }\end{array}$ & \\
& 7.0 Fr dual lumen & UVC/femoral \\
$5-15 \mathrm{~kg}$ & $7.0 \mathrm{Fr}$ dual lumen & Femoral/sub-clavian \\
$16-30 \mathrm{~kg}$ & $9.0 \mathrm{Fr}$ dual lumen & Femoral/sub-clavian \\
$>30 \mathrm{~kg}$ & 11.0 Fr dual lumen & Femoral/sub-clavian \\
\hline
\end{tabular}

UAC, umbilical arterial catheter; UVC, umbilical venous catheter

Table 2. Suggested catheters

\begin{tabular}{ll}
\hline Company & Catheter \\
\hline MedComp & Single lumen $5 \mathrm{Fr}$ \\
& Dual lumen $7 \mathrm{Fr}$ side by sidea \\
& Dual lumen $7 \mathrm{Fr}$ coaxial $^{\mathrm{b}}$ \\
& Dual lumen $9 \mathrm{Fr}^{\mathrm{a}}$ \\
& Dual lumen $9 \mathrm{Fr}^{\mathrm{a}}$ coaxial \\
& Dual lumen $6.5 \mathrm{Fr}^{\mathrm{a}}$ \\
Vas-cath & Dual lumen Minipuncture 7 Fr \\
\hline
\end{tabular}

a Not currently available

b May be high percentage recirculation, high resistance

decrease in arterial blood flow rate out of the patient with high resistance circuit back into the patient. Another access in the literature has been via a femoral arterial catheter utilizing a 4-Fr (18-gauge) arterial access with return to a 5-Fr (16-gauge) venous catheter. The size of the patient certainly will regulate the size of the access. Older children can have an arterial access of the radial artery with an 18 -gauge or 20-gauge arterial " $V$ " with return to an 18gauge or larger venous catheter. A continuous mean arterial blood pressure (MAP) of greater than $40-50 \mathrm{mmHg}$ is required for adequate blood flow rate. Patients with low MAPs will have somewhat lower blood flow rates. In this setting, increased risk of clotting or the opposite, increased risk of over heparinization, may occur. A pump-assisted CAVH(D) can improve the ultrafiltration goals in those patients with a relative low MAP [2].

\section{Access-CVVH(D)}

CVVH(D) access either by a dual-lumen venous catheter or two separate venous catheters can be utilized (Tables 1, 2). Cannulation of the femoral vein with a dual-lumen 6.5-Fr (Vascath. Mississauga, Ontario, Canada), duallumen 7-Fr (MedComp-Medical Components, Harleysville, Pa., USA) or a dual-lumen 7-Fr Minipuncture (Cook, Bloomington, Ind., USA) catheters will allow adequate blood flow rates for a pump-assisted CVVH(D) system. Because CVVH(D) is not MAP dependent, the blood flow rate will be dependent upon blood/pump speed as well as the resistance of the circuit. In larger children, roughly $15-25 \mathrm{~kg}$, access can be attained with a dual-lumen 9-Fr venous catheter via a subclavian or femoral approach. In those children greater than $25-30 \mathrm{~kg}, 11-\mathrm{Fr}$ dual-lumen catheters can be utilized (Table 1).

\section{Heparinization}

The degree of heparinization required will be dependent on: (1) coagulopathy of the patient, (2) blood flow rates, and (3) vascular access of the patient. Once the system is primed according to the manufacturers' suggestions, excluding those patients who are coagulopathic, loading with $10-20$ units $/ \mathrm{kg}$ of heparin followed by a continuous heparin infusion of 10-20 units $/ \mathrm{kg}$ per hour will give adequate anticoagulation in most patients. Assessment of adequate anticoagulation is somewhat center dependent. Experience has shown that utilization of a central laboratory measuring partial thromboplastin time is an inadequate measure of anticoagulation. This is due to the relatively long turnaround time that most laboratories require for this assay. Measurement of partial thromboplastin time or activated clotting time (ACT, Hemochron, International Technidyne, Edison, N. J., USA) at bedside makes immediate assessment of heparinization possible [20]. Normal ACTs for a non-heparinized patient range from 80 to $120 \mathrm{~s}$. Adequate heparinization occurs when the ACT distal to the hemofilter is between 150 and $200 \mathrm{~s}$. In those patients who are coagulopathic or who are at risk for bleeding, a no loading dose or a small amount (e.g., 5-10 units $/ \mathrm{kg}$ ) of heparin can be utilized at onset of renal replacement therapy (RRT). This can be followed by a "heparin-free" or low-dose heparin infusion treatment. Frequent monitoring of ACTs then will allow for "tight" control of heparinization.

Consistency of the sampling site for ACT analysis is important. ACT sampling at the arterial port proximal to the heparin infusion reflects a residual heparin effect from the patient. ACT sampling distal to the heparin infusion in the venous port reflects heparinization of the hemofilter prior to entry into the patient. Center perference dictates sampling one port versus the other, thus consistency of sampling is important.

In those patients in whom systemic heparinization must be prevented, regional heparinization can be utilized [21]. By infusing the heparin pre filter and protamine post filter (1 $\mathrm{mg}$ of protamine for every $100-200$ units of heparin infused), adequate heparinization of the hemofilter with minimal systemic heparin effect can be attained. Close observation of ACTs is important in this setting, and protamine side effects (hypotension, flushing, increased pulmonary hypertension) should be looked for.

Patients on CAVH(D) with a relatively low MAP may require more heparinization. The decreased MAP will give a relatively lower blood flow rate with an increasing chance of clotting. This is further complicated in the cardiac or neonatal population whose hematocrit $(\mathrm{Hct})$ is relatively high. The higher the Hct in this population, the higher the resistance within the circuit, thus decreasing the blood flow rate. In order to decrease the amount of heparinization used in this population, attempts to increase the MAP as well as increasing blood flow rates should be made. 
Table 3. Pediatric hemofilters

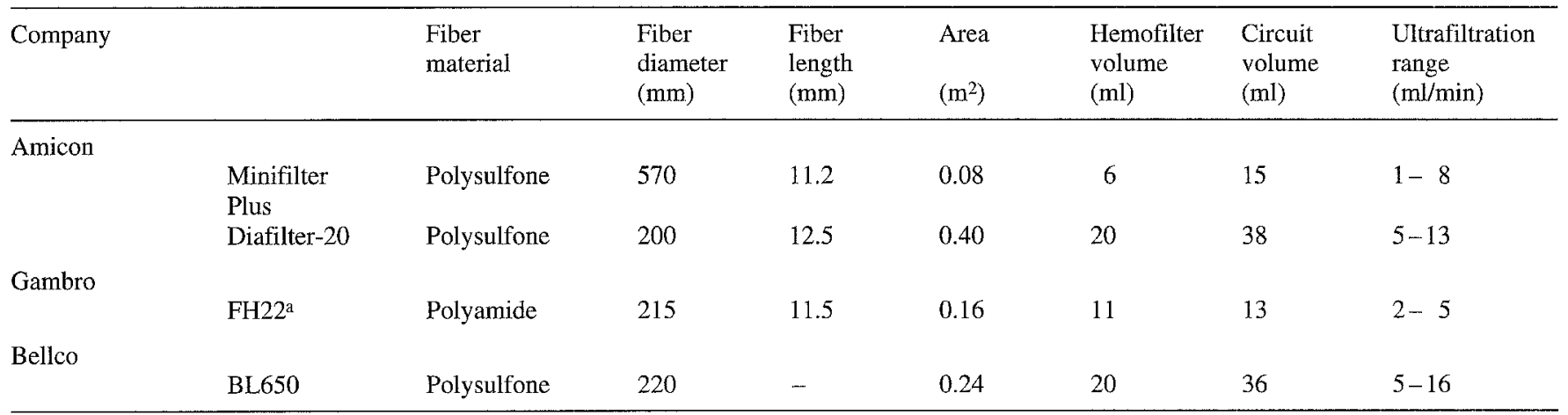

a Presently continuous arterio-venous hemofiltration only unless adapter placed available from Gambro

\section{Extracorporeal devices-CAVH(D)}

Techniques utilizing CAVH(D) are readily available. Table 3 lists currently available filters utilized for infants and the pediatric population. The common denominators of these filters include: (1) a small blood volume and (2) a relatively small surface area. Presently, not all the filters are readily available for counter current dialysis (" $D$ ") and may be used only for CAVH. Currently no veno-venous lines are included with the CAVH(D) kits.

Attention to the volume of blood extracorporeal to the patient is necessary. The total extracorporeal volume of the patient should be less than $10 \%$ unless blood priming is anticipated [22]. If blood priming is anticipated, then attention to the potassium concentration of the blood-banked blood is imperative. In some institutions, the potassium of banked blood may exceed $20 \mathrm{mEq} / 1$. Thus, in infants undergoing CAVH(D) for hyperkalemia, a "bolus" of potassium may occur if using standard blood-banked blood. An additional problem that blood priming causes is that of a high Hct with slugging of blood resulting in a decrease in blood flow. This may then start the cascade of using more heparin than necessary due to the increase in flow resistance. Diluting the blood to a Hct of $30 \%-40 \%$ with saline will decrease the potassium as well as the blood flow resistance without significant hemodynamic compromise of the patient.

\section{Blood flow rates devices-CVVH(D)}

As mentioned above, blood flow rates on CAVH(D) are related to the MAP of the patient as well as the resistance of the circuit. In children with a MAP less than 40 or 50 $\mathrm{mmHg}$, a blood flow rate of less than 5 or $10 \mathrm{ml} / \mathrm{min}$ may occur in the system with an increased risk of clotting. In patients who normally have high Hct (neonates, cyanotic heart disease, chronic pulmonary insufficiency), the combination of a low MAP and the additive effect of an increased Hct will result in a low-flow circuit with risk of clotting. During times of relatively low blood flow rate, examination of the system is necessary to insure that no clamps are partially occluding the blood flow, as well as insuring the system is not clotted. Placement of a three-way stop-cock on the arterial side of the hemofilter allows for access for flushing to exclude clotting. The risk of the three-way stop-cock is that of an accidental infusion (fluid, air) occurring as well as providing another site for disconnection or risk of infection. If examination of the systems shows no clotting, then attention to the venous acces is important. Assuring that the venous acces is of a relatively short length as well as a large bore will assure that the venous access does not give a highresistance circuit resulting in decreased blood flow rates. In patients with "tense actites" whose venous line returns to the femoral vein. theoretically an increased intra-abdominal pressure may cause high venous pressures, inhibiting the venous return from the CAVH(D) system. Potentially changing the venous access to above the diaphragm may increase the blood flow rate. In those children with relatively low MAPs, CAVH(D) may not be attainable; thus, CVVH(D) would be necessary.

\section{Extracorporeal devices-CVVH(D)}

Today there exists no easily obtainable pediatric CVVH(D) machine. The requirements for these machines are similar to those for $\mathrm{HD}$, including attention to varying blood flow rates (as little as $10-50 \mathrm{ml} / \mathrm{min}$ ) and to small extracorporeal volume. Hospal (Cobe-Gambro-Hospal, Lakewood, Colo., USA) markets a CVVH(D) system with the smallest extracorporeal lines (pediatric lines) of $94 \mathrm{ml}$. The addition of a hemofilter may add between 6 and $20 \mathrm{ml}$ of volume. Utilizing this system, blood flow rates can range from 10 to $700 \mathrm{ml} / \mathrm{min}$, depending upon the vascular resistance of the lines as well as of the need of the patient. A blood flow rate beyond $300 \mathrm{ml} / \mathrm{min}$ results in no improvement in dialysis or ultrafiltration efficiency due to the limitations of the hemofilter surface area as well as blood flow resistance.

At the University of Michigan, as well as other locations, we have adapted a Gambro AK-10 HD machine (Cobe-Gambro-Hospal, Lakewood, Colo., USA) for utilization of CVVH(D). The advantage of the system allows one to use existing blood lines available for this machine, 
and, as the patient size varies, extracorporeal volume can vary depending on the blood lines. Utilizing these lines, circuit volumes (neonatal lines plus the minifilter plus) may be as low as $38 \mathrm{ml}$. Blood flow rates utilizing this system can range from $10-$ to $300 \mathrm{ml} / \mathrm{min}$ depending upon the vascular resistance of the lines as well as of the needs of the patient. Using the formula of $80 \mathrm{ml} / \mathrm{kg}$ to calculate intravascular blood volume in infants of less than $10 \mathrm{~kg}$, CVVH(D) utilizing the AK-10 can be achieved without blood priming in children down to $5 \mathrm{~kg}$ and in the Hospal system $11 \mathrm{~kg}$.

A significant advantage of any CVVH(D) system is that of controlled blood flow rate. In those children with low MAPs as well as high Hcts, we have already noted that adequate blood flow is difficult. By using a pump-assisted system, one can easily maintain stable blood flow, resulting in steady dialysis as well as the decreased heparinization needed for the patient. Furthermore, the advantage of a CVVH(D) system is the lack of a requirement for arterial cannulation. We have routinely utilized dual-lumen venous catheters in this setting, thus requiring a single stick to the patient.

When one looks at CAVH(D) or CVVH(D), it is important to know that air emboli are potentially an issue. Utilizing this CVVH(D) system, an air-leak detector is currently in line with a venous pressure monitor for the AK-10. The venous pressure monitor further allows us to detect any early venous resistance that is seen with clotting. Because the CAVH(D) system does not utilize either an air-leak detector or a venous pressure monitor, potential risk for air emboli as well as increased clotting exists.

A recent experience at the University of Michigan, looking at the average life of a CAVH(D) system versus a CVVH(D) system, shows that in infants our average CAVH(D) system has to be changed every $12-18 \mathrm{~h}$. On CVVH(D), our average systems are changed every 2-3 days. Thus, there is a decrease in technician and nursing hours as well as a decrease in utilization of supplies for $\mathrm{CVVH}(\mathrm{D})$.

A disadvantage of CVVH(D) is that it needs constant attendance by nursing staff. Many intensive care nurses are trained to troubleshoot the CVVH(D) systems, with hemodialysis nursing staff as back-up. With the safeguards of venous and arterial alarms and air-leak detectors, this can safely be achieved by skilled nursing.

\section{Blood flow rates-CVVH(D)}

BFR in HD are usually $3-4 \mathrm{ml} / \mathrm{kg}$ per min for $3-4 \mathrm{~h}$ in order not to cause dialysis disequilibrium due to significant drops in the blood urea nitrogen (BUN). In most patients utilizing CVVH(D), urea clearance moment to moment is not that rapid [3,5]. Normal blood flow rates for CVVH(D) are not established. Our approach is to utilize between 5 and $10 \mathrm{ml} / \mathrm{kg}$ per min as long as the venous resistance is low and the BUN is not more than $100 \mathrm{mg} / \mathrm{dl}$. This will allow for adequate blood flow for ultrafiltration as well as decrease in the amount of heparin utilized.

\section{Ultrafiltration rates}

Whether one utilizes CAVH(D) or CVVH(D), the degree of ultrafiltration will be dependent upon the Hct, the blood flow rate, the efficiency of the filter (Table 3), and the pressure change at the level of the filter [transmembrane pressure (TMP)]. In patients with high Hcts, the amount of ultrafiltration will decrease as the degree of hemoconcentration increases. This is due to the vascular resistance (again affecting blood flow) as well as the amount of ultrafiltration required. With decreasing blood flow rate, if ultrafiltration occurs at a fixed rate, then the degree of hemoconcentration that occurs at the venous side of the hemofilter will increase. This may result in localized clotting, which transiently will increase the TMP, with a transient increase in ultrafiltration, but ultimately will result in loss of the system.

Maneuvers to increase the TMP include: (1) partially occluding the venous blood line, (2) increase in vertical distance between the ultrafiltration port and collection bag (this will increase the negative pressure in the ultrafiltration line), or (3) suction on the ultrafiltration port [22].

Occlusion of the venous port will further decrease blood flow setting up a cascade for clotting. Wall suction on the ultrafiltration port may hemoconcentrate to such a severe degree that clotting may begin. Furthermore, this maneuver may cause ultrafiltration to such a severe degree that it would result in hemodynamic instability. Increasing the vertical distance between a gravity drainage system and the ultrafiltration port appears to be the most "gentle" with fewer hemodynamic as well as hemoconcentration effects.

\section{Ultrafiltration devices}

Most centers have the opposite problem of controlling ultrafiltration at a steady rate ensuring that ultrafiltration is not too rapid. A hospital intravenous pump brand (IMED or IUAC) can be utilized in this setting to inhibit the ultrafiltration rate at a set rate. The ultrafiltration port is connected to regular IV tubing which is then inserted into the IMED/IV AC and drained into a volumetric collection bag. By using the pump and dialing in the prescribed ultrafiltration rate, close and accurate ultrafiltration can result. Caution, assuring that you are not attempting to draw off too much, is important. In this setting, the IMED/IV AC occlusion alarm will sound to warn the operator that insufficient ultrafiltrate is being removed. Furthermore, utilizing this system, if the CAVH(D)/CVVH(D) system is clotting and losing permeability, then the occlusion alarm on the IMED/IUAC will notify the operator that this is a potential problem. The accuracy of these pumps is somewhat variable, therefore, draining into a volumetric drainage bag will serve as a double check of the amount of ultrafiltration occurring $[23,24]$.

An ultrafiltration device is available (EQUALine fluid control system, Medica, Medolla, Italy) that operates by gravity flow for either countercurrent dialysis or for filtered replacement fluid. The variation of ultrafiltrate as well as infusion is from 0 to $999 \mathrm{ml} / \mathrm{min}$. Limited experience with this device is available in the pediatric population. 
Table 4. Urea clearance in the Minifilter Plus (blood flow rate of $50 \mathrm{ml} / \mathrm{min}$ )

\begin{tabular}{ll}
\hline $\begin{array}{l}\text { Dialysate flow rate } \\
(\mathrm{ml} / \mathrm{min})\end{array}$ & $\begin{array}{l}\text { Urea clearance } \\
(\mathrm{ml} / \mathrm{min})\end{array}$ \\
\hline 5 & 6 \\
10 & 7 \\
15 & 8 \\
20 & 9 \\
\hline
\end{tabular}

\section{Clearance}

Clearance (e.g., urea) is related to the surface area of the hemofilter, blood flow rate, and the dialysate composition and flow rate. In HD the fixed parameters are the dialysate composition (except for sodium variation machines, potassium and phosphorus supplementation) and dialysate flow rates $(500 \mathrm{ml} / \mathrm{min})$. The variables include surface area of the dialyzer (usually picked for staying within the $10 \%$ extracorporeal volume rule) and blood flow rates (which are usually in the range of $3-5 \mathrm{ml} / \mathrm{kg}$ per min). Therefore, by choosing an appropriate surface area dialyzer and setting blood flow rate at $3 \mathrm{ml} / \mathrm{kg}$ per min, a known quantity of solute (e.g., urea) is removed.

In CAVH(D)/CVVH(D) the surface areas are fixed (due to few choices, unless one increases the extracorporeal volume), and blood flow rate may be MAP dependent [especially in CAVH(D), whereas in CVVH(D) the blood flow rate may parallel the blood flow rate of HD], and the dialysate flow rate is significantly less than the dialysate in HD. If one utilizes an IMED/IV AC for infusion of dialysate and removal of ultrafiltrate, the usual maximal dialysate flow rate per single pump is $1 \mathrm{l} / \mathrm{h}$. This is contrast to HD dialysate flow rates of $30 \mathrm{l} / \mathrm{h}$. Therefore, when compared with HD, the clearance efficiency of CAVH(D)/CVVH(D) is not due to hourly clearance but due to its continuous nature. Little data exist on clearance of solute in CAVH(D)/CVVH(D) in the pediatric population with the blood flow rates and dialysate flow rates typically used in infants and children (Table 4) [25]. Studies at the University of Michigan are ongoing in order to identify optimal blood flow rates in relationship to dialysate flow rates.

The other approach to clearance is high ultrafiltration with replacement with a filtered replacement fluid (FRF) [1]. This entails placing a physiological solution pre or posthemofilter at a fixed rate and ultrafiltrating at a similar rate in order to remove solute by passive diffusion as opposed to countercurrent dialysis. A four-bag system of four separate components of this physiological solution is spiked into a multipronged solution set that mixes equally into a single solution. The advantage of this approach is in hemofilters that are primarily for CAVH/CVVH not CAVH(D)/CVVH(D). The disadvantage of this system is that it is somewhat cumbersome and, due to its four-bag design, increases the risk of error if all bags do not drain equally. To date no data exist comparing clearance by FRF versus ' $D$ ' in CAVH(D)/CVVH(D). FRF logically may have a better clearance due to the high potential turnover of ultrafiltrate.
Table 5. Composition of a typical peritoneal dialysis (PD) solution and homemade solution

\begin{tabular}{|c|c|c|}
\hline & $\begin{array}{l}\text { PD } \\
\text { solution }\end{array}$ & $\begin{array}{l}\text { Homemade } \\
\text { solution }\end{array}$ \\
\hline Glucose & $1.5 \%, 2.5 \%, 4.25 \%$ & 0 \\
\hline $\mathrm{Na}(\mathrm{mEq} / \mathrm{l})$ & 132 & $130-145$ \\
\hline $\mathrm{Cl}(\mathrm{mEq} / \mathrm{l})$ & 96 & $94-109$ \\
\hline $\mathrm{Ca}(\mathrm{mEq} / \mathrm{l})$ & 3 & $3-4$ \\
\hline $\mathrm{Mg}(\mathrm{mEq} / \mathrm{l})$ & 0.5 & 0.5 \\
\hline $\mathrm{K}(\mathrm{mEq} / \mathrm{l})$ & $0-5^{a}$ & $0-5$ \\
\hline Phosphate (mg/dl) & 0 & 0 \\
\hline Lactate $(\mathrm{mEq} / 1)$ & 40 & 0 \\
\hline Bacarbonate $(\mathrm{mEq} / \mathrm{l})$ & 0 & $30-40$ \\
\hline
\end{tabular}

$\mathrm{Na}$, Sodium; $\mathrm{Cl}$, chloride; $\mathrm{Ca}$, calcium; $\mathrm{Mg}$, magnesium; $\mathrm{K}$, potassium

a Normally is 0 , may be added to normal PD solution

With CVVH(D) at higher blood flow rates, potassium as well as phosphorus clearance can be adequately obtained by ' $D$ '. Studies are in progress at the University of Michigan to evaluate this. The debate over the utilization of FRF as predilution versus postdilution is ongoing. The position of FRF infusion relative to the hemofilter remains an individual preference. Proponents of predilution advocate this route due to less hemoconcentrating occurring distally on the hemofilter. No specific pediatric studies to date have adequately addressed this issue.

\section{Dialysate composition}

In those patients undergoing CAVH(D) or CVVH(D) dialysis can occur either via a countercurrent system at the level of the hemofilter or via a FRF solution. Utilizing the countercurrent system, the standard solution used by most centers is that of dialysate utilized in PD. Because the ultrafiltration that occurs with a hemofilter is not glucose dependent (as opposed to patients on PD), the concentration of glucose in the PD solution is irrelevant. Table 5 lists the composition of a typical PD solution to which one can easily add potassium up to 4 or $5 \mathrm{mEq} / \mathrm{l}$ if necessary. As it has been shown with HD, the lactate in the PD solution may result in a metabolic acidosis of the patient. Our experience with infants as well as patients with hepatic insufficiency leads us to utilize a "homemade" solution for countercurrent dialysis (Table 5). The difference between this solution and that of PD is the substitution of the bicarbonate as a well as potentially higher dialysate sodium. Concern of calcium precipitation has been raised in utilizing this type of solution, yet studies with bicarbonate added to standard PD solution have shown no degree of calcium precipitation or drop in the calcium in the PD bath [26]. Furthermore, we have increased the calcium in the solution up to $4 \mathrm{mEq} / 1$ without obvious precipitation with bicarbonate and with noted improved calcium levels in the patient. The disadvantage of this system is that phosphorus cannot be added to the system due to the risk of precipitation with the calcium. Using the ' $\mathrm{D}$ ' approach, one then infuses dialysate countercurrent to the blood flow of the hemofilter. 
Table 6. Composition of the filtered replacement fluid

\begin{tabular}{ll}
\hline IV “A" & $1000 \mathrm{ml}$ of $0.9 \mathrm{NS}+7.5 \mathrm{ml}$ of $\mathrm{CaCl}_{2}(10.35 \mathrm{mEq})$ \\
IV "B" & $1000 \mathrm{ml}$ of $0.9 \mathrm{NS}+1.6 \mathrm{ml}$ of $50 \% \mathrm{MgSO}_{4}(6.4 \mathrm{mEq})$ \\
IV "C" & $1000 \mathrm{ml}$ of $0.9 \mathrm{NS}$ \\
IV "D" & $1000 \mathrm{ml} \mathrm{D} 5 \mathrm{~W}+150 \mathrm{mEq} \mathrm{NaHCO}$ \\
\hline
\end{tabular}

NS, Normal saline; $\mathrm{CaCl}_{2}$, calcium chloride; $\mathrm{MgSO}_{4}$, magnesium sulfate; $\mathrm{NaHCO}_{3}$, sodium bicarbonate

If one utilizes the FRF approach to enhance clearance, then the composition of the FRF must remain physiological (Table 6). This has the same disadvantage of the 'D' solution in that no phosphorus exists in the bath due to the risk of calcium precipitation. Furthermore, this contains glucose (as opposed to our homemade solution), which in some circumstances may induce hyperglycemia. Therefore, adjustment of the glucose or addition of insulin may be necessary with this approach.

\section{CAVH(D)/CVVH(D) on extracorporeal membrane oxygenation}

Extracorporeal membrane oxygenation (ECMO) is now commonly utilized for support of children with reversible pulmonary and cardiac failure. Renal insufficiency occurs in a significant percentage of children on ECMO who can be well managed by CAVH(D)/CVVH(D) that is placed directly into the system. The resistance of the CAVH(D)/CVVH(D) filter is less than that of the oxygenator of the ECMO circuit. This must be taken into account when the hemofilter is added to the system in order not to cause "stealing" of blood flow away from the oxygenator [27].

When blood flow is measured through the hemofilter, the blood flow rate is in the range of $80-150 \mathrm{ml} / \mathrm{min}$. In a small infant (e.g., $3 \mathrm{~kg}$ ), this could result in a hemofilter blood flow of up to $50 \mathrm{ml} / \mathrm{kg}$ per min (as opposed to HD of $3-4 \mathrm{ml} / \mathrm{kg}$ per $\mathrm{min}$ ). This will impact significantly upon clearance of solute with the dialysate flow rate having less impact. Hoffman clamps (small "C" clamps) may be added to the "arterial" side of the hemofilter in order to decrease the blood flow rate into the hemofilter if necessary [27].

\section{Ultrafiltration}

If ultrafiltration occurs alone without dialysis, the attention to the contents of the ultrafiltrate is important. Isolated ultrafiltration (known as SCUF-slow continuous ultrafiltration) is well tolerated in larger individuals. In hemodynamically unstable and small children, SCUF is less well tolerated due not only to fluid but also osmolar shifts. As an example, if a 3-kg infant loses $500 \mathrm{ml}$ of ultrafiltrate, then the contents of the ultrafiltrate will include solute based on sieving coefficients. Sodium, as an example, will come across as a $1: 1$ concentration of plasma to ultrafiltrate. Thus, this infant could loose $70 \mathrm{mEq}$ of sodium (assuming a serum sodium of $140 \mathrm{mEq} / \mathrm{l}$ ). The resulting osmolar shift could have significant hemodynamic impact upon this child. By utilizing either countercurrent dialysis or FRF, fewer osmolar shifts occur resulting in a more stable treatment.

\section{Ultrafiltration with dialysis}

Many centers utilize IMED/IV AC IV pumps for installation of dialysate as well as control of the ultrafiltration. Thus, if one needed to run dialysate at $200 \mathrm{ml} / \mathrm{h}$, yet wanted a net ultrafiltration of $50 \mathrm{ml} / \mathrm{h}$, the infusion IMED/IV AC would be set at 200 while the ultrafiltration IMED/TV AC would be set at $250 \mathrm{ml}$. A urometer bag is placed at the final collection port on the ultrafiltration IMED/IV AC. This amount of fluid is recorded hourly and double checked against the IMED/IVAC pump. Experience at the University of Michigan shows that, with relatively small dialysate flow rates of $100-300 \mathrm{ml} / \mathrm{h}$, there is minimal variance noted using the system. When one increases dialysate flow rates to 500 or $700 \mathrm{ml} / \mathrm{h}$, increased variances of the IMED/IV AC system are noted.

\section{Unique issues of CAVH(D)/CVVH(D)}

In HD, due to the relatively rapid turnover of blood as well as the warming of dialysate (with most HD having adjustable dialysate bath warmers), cooling of the infant or child is unusual. Because of the relatively slow blood flow rate [as in CAVH(D)] with no active warming of the dialysate, cooling of the child is common and should be anticipated. Measures such as a warming blanket or an overhead warmer must be available for the patient.

As opposed to HD, the biocompatibility of the hemofilter membranes has little activation of complement [28]. Therefore, minimal effect of leukocyte and platelet aggregation which occurs in the vascular space (and in the lung increasing the arterial-alveolar gradient) results in minimal increase in oxygen demands on an severely ill child.

\section{Drug removal}

Depending upon the sieving coefficient as well as the dialysis efficiency (combination of blood flow rate, dialysate flow rate, and the surface area of the filter), drug removal will vary. The sieving coefficient of drug removal by SCUF alone is well known [1]. Because the clearance of solute or drugs is blood and dialysate flow rate dependent, attention to drug levels is important in order not to overdose or underdose patients. Placement of drugs (e.g., vancomycin) in the dialysis (not FRF) could theoretically result in steady-state levels. This is not well tested and is presently not recommended.

\section{Summary}

CAVH(D)/CVVH(D) are now commonly utilized and are additional therapies for RRT in the pediatric population. It is best used in the hemodynamically unstable patient and in 
those patients needing constant ultrafiltration (as in patients with oliguria needing medication and/or nutrition). For clearance, moment to moment, HD and PD are superior, yet due to its persistent nature, CAVH(D)/CVVH(D) techniques have their place in the field of RRT. The techniques of CAVH(D)/CVVH(D) are relatively easy to carry out, with CAVH(D) having the advantage of simplicity. At the University of Michigan we have preferred to utilize CVVH(D) due to its ease of application, independent of the MAP or Hct, less frequent replacement of supplies, and reduced heparin usage in the patient. Individual preference should dictate the method which is best suited for the patient.

Acknowledgements. The authors would like to thank Ms. Sharon Doyle for her diligence in preparation of this manuscript and the HD and intensive care nurses at both institutions for their excellent care of the children.

\section{References}

1. Golper TA (1985) Continuous arteriovenous hemofiltration in acute renal failure. Am J Kidney Dis 6: 373-386

2. Yorgin PD, Krensky AM, Tune BM (1990) Continuous venovenous hemofiltration. Pediatr Nephrol 4: 640-642

3. Zobel G, Ring E, Zobel V (1989) Continuous arteriovenous renal replacement systems for critically ill children. Pediatr Nephrol 3: $140-143$

4. Liberman KV (1987) Continuous arteriovenous hemofiltration in children. Pediatr Nephrol 1:330-338

5. Zobel G, Ring E, Trop M, Stein JI (1986) Arteriovenous hemodiafiltration in children. Inf. J Pediatr Nephrol 7: 203-206

6. Bishif NA, Welch TR, Strife FS, Ryckman FC (1990) Continuous hemodiafiltration in children. Pediatrics 85: 819-823

7. Nevins TE, Mauer SM (1993) Infant hemodialysis. In: AR Nissenson and RN Fine (eds) Dialysis therapy, 2nd edn. Hanley and Belfus, Philadelphia, pp 349-353

8. Fine RN, Salusky I (1986) CAPD/CCPD in children: fours years' experience. Kidney Int 30: S7-S10

9. Assadi FK (1988) Treatment of acute renal failure in an infant by continuous arteriovenous hemodialysis. Pediatr Nephrol 2: 320-322

10. Ronco C, Brendolan A, Bragantini L, Chiaramonte S, Feriani M, Fabris A, Dell'Aquila R, LaBreca G (1986) Treatment of acute renal failure in newborns by continuous arterio-venous hemofiltration. Kidney Int 29: $908-915$

11. Liberman KV, Nardi L, Bosch JP (1985) Treatment of acute renal failure in an infant using continuous arteriovenous hemofiltration. J Pediatr 106: 646-649
12. Loene MR, Jenkins RD, Golper TA, Alexander SR (1986) Early experience with continuous arteriovenous hemofiltration in critically ill pediatric patients. Crit Care Med 14: $1058-1063$

13. Heney D, Essex-Carter A, Brocklebank JT, Bailey CC, Lewis IJ (1990) Continuous arteriovenous haemofiltration in the treatment of tumour lysis syndrome. Pediatr Nephrol 4: 245-247

14. Thompson GN, Butt WW, Shann FA, Kirby DM, Henning RD, Howells DW, Osborne A (1991) Continuous venovenous hemofiltration in the management of acute decompensation in inborn errors of metabolism. J Pediatr 6: 879-884

15. Bargman JM (1990) Complications of peritoneal dialysis related to raised intra-abdominal pressure. Dial Transplant 19: 70-96

16. Bunchman TE, Meldrum MK, Meliones JE, Sedman AB, Kershaw DB (1992) Pulmonary function variation in ventilator dependent critically ill infants on peritoneal dialysis. Adv Perit Dial 8: 75-78

17. Bunchman TE, Wood EG, Lynch RE (1987) Hydrothorax as a complication of pediatric peritoneal dialysis. Peritoneal Dial Bull 7 : $237-239$

18. McClung MR (1983) Peritonitis in children receiving continuous ambulatory peritoneal dialysis. Pediatr Infect Dis $\mathbf{J} 2: 328-331$

19. Jenkins RD, Kuhn RJ, Funk JE (1988) Clinical implications of catheter variability on neonatal continuous hemofiltration. Trans Am Soc Artif Intern Organs 34: 108-111

20. Geary DF, Gajaria M, Fryer-Keene S, Willumsen J (1991) Low dose and heparin-free hemodialysis in children. Pediatr Nephrol 5: $220-224$

21. Kaplan AA, Petrillo R (1987) Regional heparinization for continuous arterio-venous hemofiltration. Trans Am Soc Artif Intern Organs 33: $312-315$

22. Liberman K (1993) Continuous arteriovenous renal replacement in children. In: AR Nissenson and RN Fine (eds) Dialysis therapy, 2nd edn. Hanley and Belfus, Philadelphia, pp 373-378

23. Jenkins R, Harrison H, Chen B, Arnold D, Funk J (1992) Accuracy of intravenous infusion pumps in continuous renal replacement therapies. ASAIO J 38: 808-810

24. Roberts M, Winney RJ (1992) Errors in fluid balance with pump control of continuous hemodialysis. Int J Artif Organs 15: 99-102

25. Technical Data (1991) Amicon Division, W. R. Grace, Beverly

26. Bunchman TE, Ballal SA (1991) Treatment of inflow pain by $\mathrm{pH}$ adjustment of dialysate in peritoneal dialysis. Peritoneal Dial Int 11 : $179-180$

27. Remenapp RT, Copenhaver WG, Chapman RA, Bartlett RH (1992) A reliable method to determine the amount of hemofilter shunt. Proceedings of the 8th Annual Children's National Medical Center ECMO Symposium, Breckinridge, Colorado, February 1992. Abst Book, pp 33

28. Craddock PR, Fehr J, Brigham KL, Kronenberg RD, Jacob HS (1987) Complement and leukocyte-mediated pulmonary dysfunction in hemodialysis. N Engl J Med 296: 769-774 